\title{
Endoscopic colloid cyst resection by puncture of the medial wall of lateral ventricle, posterior to the foramen of Monro
}

\author{
D. Păunescu ${ }^{1}$, M. Gorgan, Ligia Tătăranu \\ "Bagdasar-Arseni" Clinic Emergency Hospital, Bucharest, Romania \\ ${ }^{1} \mathrm{PhD}$ Student in Neurosurgery, "Carol Davila" University of Medicine and \\ Pharmacy Bucharest, Faculty of Medicine, Departament of Neurosurgery
}

\begin{abstract}
No consensus among neurosurgeons has been reached so far concerning optimal therapeutic strategy for colloids cysts, especially as these are non-invasive,slowgrowing, benign lesions. In symptomatic cases, endoscopic resection can be achieved as first intention when it seems to have a cyst containing fluid and there is ventriculomegaly. Cyst location and degree of superior distension of the third ventricle roof, associated with the emergence of Monro's foramen can cause problems when using a rigid endoscope, the visualisation being impossible or difficult. Posterior interventricular foramen puncture may be helpful in such cases.
\end{abstract}

Keywords: colloid cyst, endoscopy

\section{Introduction}

Colloids cysts are sow growing benign tumors, but the risk of neurological deterioration or sudden death causes therapeutic indication in symptomatic cases. Endoscopic surgery is performed to ensure a normal circulation of cerebrospinal fluid and to resect the colloid cyst via a minimally invasive approach.

\section{Case presentation}

A man of 52 years was admitted to the Neurosurgery Clinic of the Emergency Clinic Hospital "Bagdasar-Arseni"
Bucharest, for intermittent headache and impaired recent memory, symptoms appeared about 3 months before presentation and gradually accelerated.

Clinical examination revealed no focal neurological signs, but loss of recent memory was present. Eye examination revealed mild bilateral papilledema.

Preoperative CT scan revealed an expansive round shape intraventricular process with a diameter of $20 \mathrm{~mm}$, inserted in the ceiling of the third ventricle, immediately dorsal to the foramen of Monro. It was a discrete heterogeneous hyperdense mass (with a high density core located to the rear with a diameter of 10 $\mathrm{mm}$ ) and it did not show contrast enhancement. Biventricular hydrocephalus was also present. Preoperative multiplanar MRI clearly showed the cyst location and bilateral obstruction of the foramen of Monro, leading to secondary hydrocephalus (symmetric increased volume of lateral ventricles and the signs of transependymal resorption of cerebrospinal fluid). A wellshaped heterogeneous mass was obstructing the third ventricle. On MRI, the tumor was isodense with an eccentric hyperdense core on T1-weighted images and hyperdense with a hypointense core on T2-weighted images (Figure 1). 

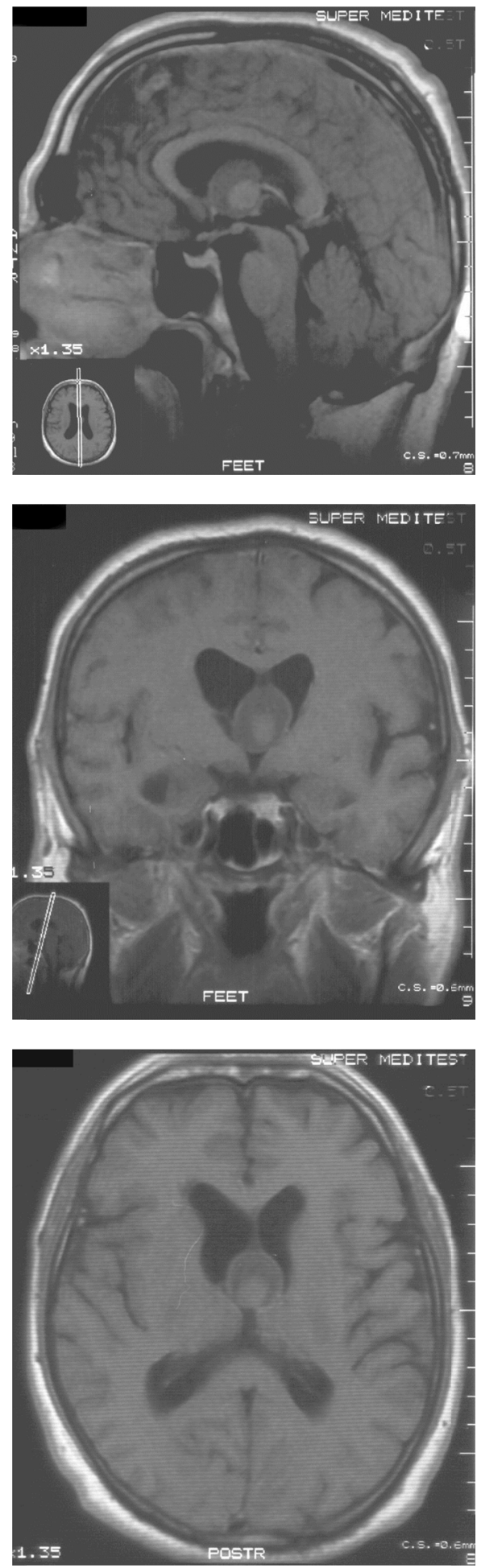

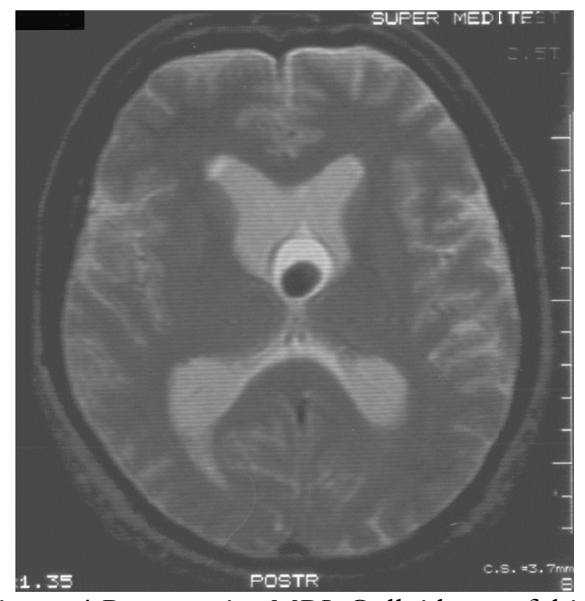

Figure 1 Preoperative MRI. Colloid cyst of third ventricle and bilateral obstruction of the Monro's foramina with secondary hydrocephalus

After discussing the risks and benefits of various treatment options with the patient we recommended endoscopic resection of the cystic mass. Necessary equipment for conventional surgery was prepared in case of failure of the endoscopic procedures.

The technique was performed under general anesthesia. A $30^{\circ}$ rigid Decq neuroendoscop was used (Karl Storz GmbH \& Co, Tuttlingen, Germany), whose channels allowed the introduction of instruments with a diameter of $1.7 \mathrm{~mm}$ and $3 \mathrm{~mm}$. The procedure consisted of an endoscopic transcortical - transventricular approach: the introduction of the neuroendoscope through a frontal burr hole into the lateral ventricle, initial monopolar coagulation posterior to the foramen of Monro, puncture and manual aspiration with a needle and opening of the cyst's wall with microscissors and forceps so that large aspirating probes could be used. Cyst location and degree of distension of the third ventricle roof imposed a puncture posterior to the Monro foramen. The cyst was completely obstruating the interventricular foramen. Its content could be partially aspirated, although the viscosity was increased. A solid, hard intracystic area 
with microcalcifications was found and removed. Small membrane remains attached to the roof of the third ventricle and to the internal cerebral veins were coagulated. Complete resection of the cyst with bilateral desobstruction of Monor's foramen was achieved. (Figure 2). The operating time was 110 minutes. Pathologic examination confirmed the diagnosis of colloid cyst.

Postoperative evolution was favorable, with partial remission of symptoms (some degree of recent memory disorders persisted). The patient was discharged in good neurological condition in the $7^{\text {th }}$ postoperative day.

At the $3^{\text {rd }}$ month follow-up visit the patient did not show any focal neurological deficits and memory disorders were remitted. His fundoscopic examination was normal. MRI brain images revealed total resection of colloid cyst and hydrocephalus resolution (Figure 3). Two years after surgery there were no signs of recurrence.

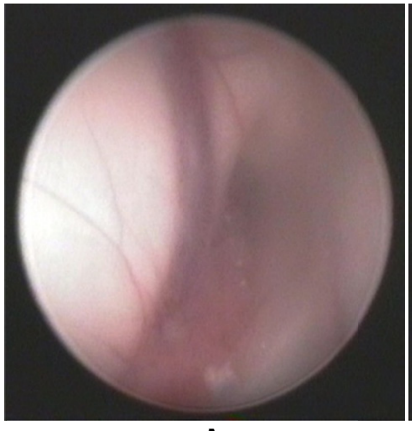

A

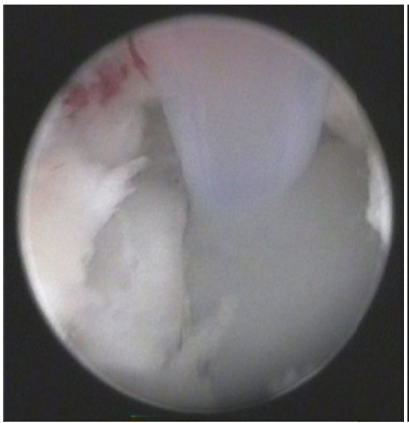

D

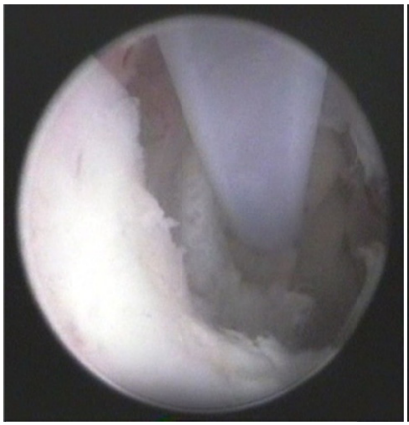

G

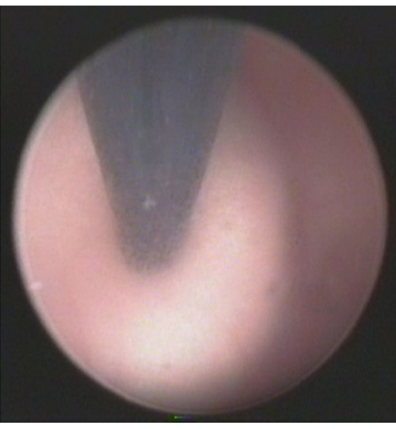

B

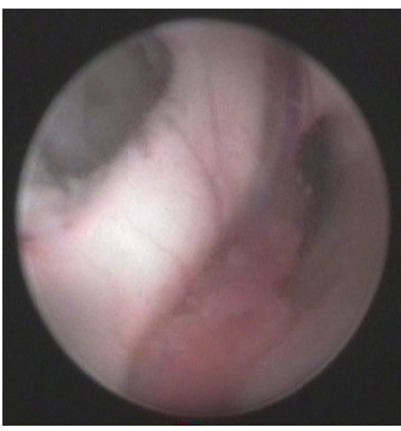

E

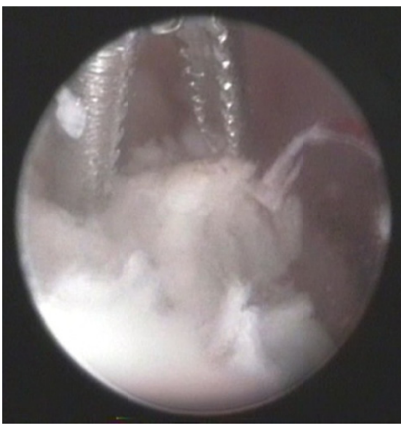

$\mathbf{H}$

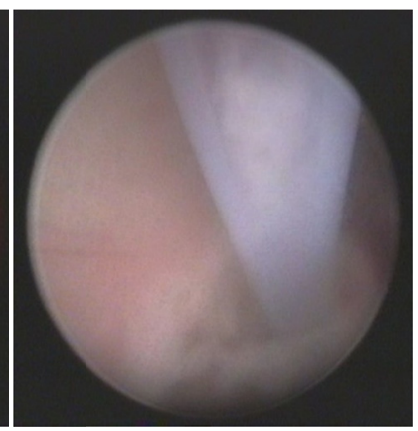

C

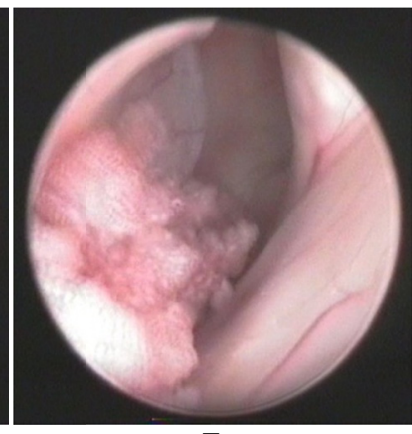

$\mathbf{F}$

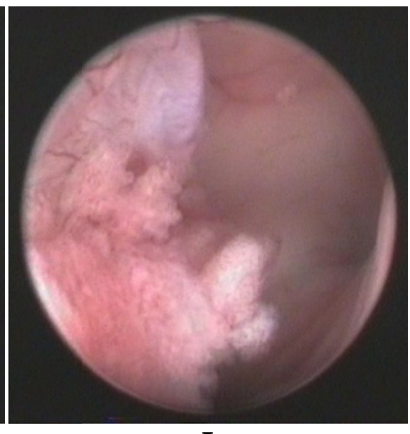

I 


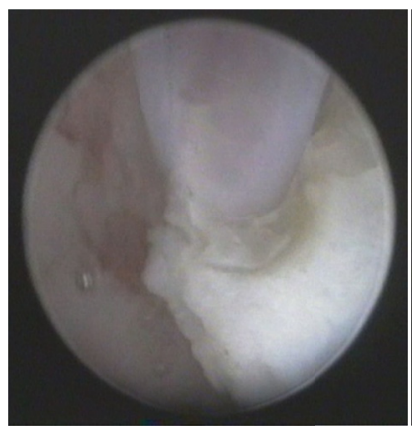

$\mathbf{J}$

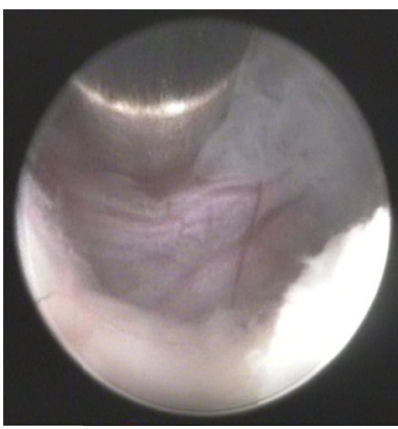

$\mathbf{K}$

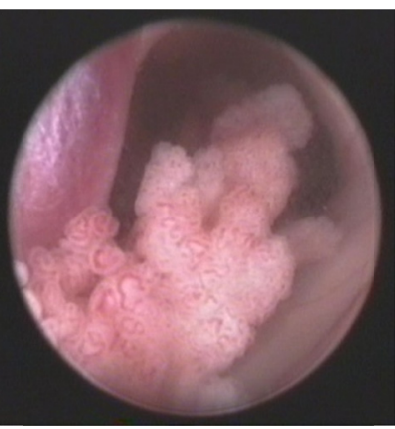

L

Figure 2 Endoscopic intraoperatory images. Resection of a colloid cyst of the third ventricle. A). The cyst obsructs the Monro's foramen, pushing the medial wall of the lateral ventricle. B). Perforation of the medial walll of the lateral ventricle by coagulation posterior to the foramen of Monro. C), D). Needle aspiration. E), F).

Partial desobstruction of the foramen. G). Cystic drainage H). Removal of the solid area using a forceps. I).

Widened Monro's foramen, allowing the visualization of the interior of the third ventricle. J). Removal of the

solid parts K). Coagulation of the cystic membranes. L). Complete desobstruction of the Monro's foramen
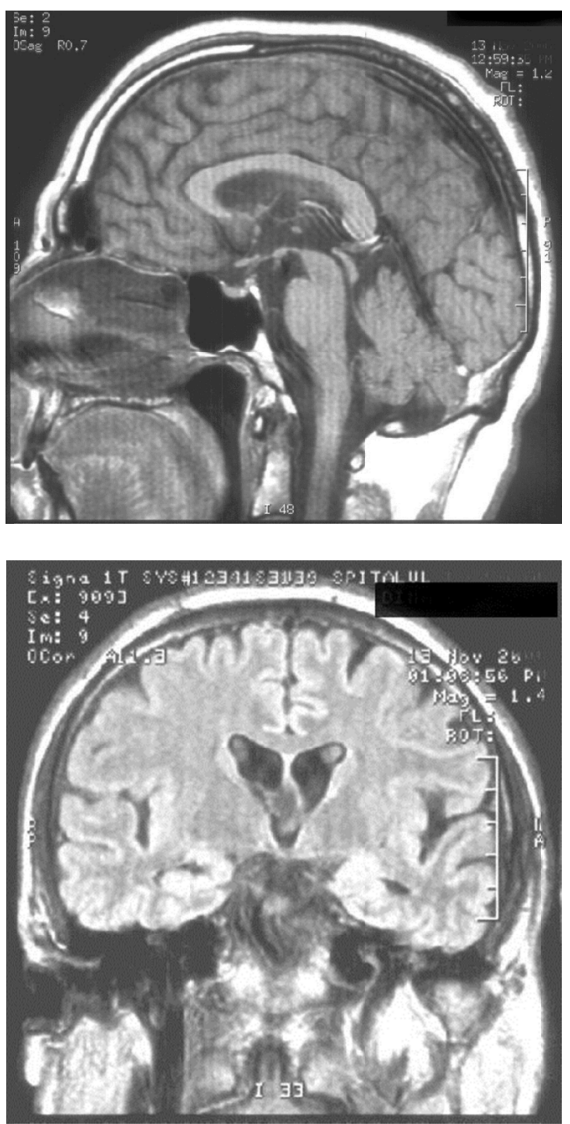

Figure 3 Postoperative MRI images. Complete endoscopic removal of third ventricular colloid cyst and restore CSF flow into the third ventricle

\section{Discussion}

Colloids cysts are benign tumors usually located in the anterior roof of the third ventricle between the columns of the fornices and are frequently attached to the stroma of the choroid. It is believed to be of endodermal origin from a vestigial third ventricular structure ( the paraphysis) and is rarely associated with craniofaringioma. The cyst may be pendulous and cause intermittent CSF obstruction with a ballvalve mechanism. It is filled with mucus that on CT may be either hyperdense (two thirds) or hypodense (one third). There are no calcifications and there is usually enhancement of the cyst wall.

Although rare, they raise neurosurgical interest because these epithelium-lined cysts can cause serious morbidity that may lead to acute obstructive hydrocephalus, increased intracranial pressure and, rarely, intracystic hemorrhage. In the absence of treatment there is a considerable risk of neurological damage or sudden death.

Cysts colloids represents $0.2-2 \%$ of brain tumors and less than $1 \%$ of symptomatic brain tumors $(2,4,3,5,12$, 
DOI: 10.2478/v10282-012-0004-3

14, 17, 19). Although these tumors are considered congenital, their presentation in childhood is rare. The tumors are usually symptomatic in patients aged $20-50$ years (21). Over $99 \%$ of colloids cysts develop in the third ventricle, in most cases in the anterior roof.

Due to anatomical location, colloid cysts of the third ventricle usually cause obstruction of Monro foramen, leading to mono or biventricular hydrocephalus, with a more or less rapidly evolution, sometimes paroxysmal. The existence of hydrocephalus determines the clinical symptoms.

Typically, colloid cysts are found incidentally. Some colloid cysts cause no signs or symptoms at all, and are only discovered when a patient has a neuroimaging exploration for unrelated reasons. If symptomatic, they are associated with paroxysmal headache associated with changing head position considered to be the consequence of intermittent obstruction of the interventricular foramen or aqueduct of Sylvius by pedunculated lesion suspended from the roof of the third ventricle $(16,17)$. More commonly, patients present with progressively installed headache sometimes associated with other symptoms of intracranial hypertension: nausea, vomiting, altered consciousness, papilledema, blurred vision, diplopia, etc. Sometimes symptoms occur or get worse paroxysmal. Typically seen are "drop attacks": sudden spontaneous falls while standing or walking without loss of consciousness caused by decreased leg muscle tone.

Though colloids cysts are benign, slowgrowing tumorsthe risk of sudden neurologic deterioration or sudden death lead to surgical treatment in symptomatic cases. In asymptomatic cases, although neurological deterioration is possible in a small number of cases we cannot identify high-risk patients. Therefore, the mere surveillance of these cases would be justified only in the elderly, in those with low life expectancy or those with increased anesthetic risk (17). In other cases, the treatment seems the most prudent solution and is agreed by most neurosurgeons.

Several therapeutic options have been proposed: cerebrospinal fluid diversion, stereotactic aspiration, conventional microsurgical intervention and endoscopic surgery.

Endoscopic surgery uses the same path to the Monro's foramen as transcortical approach but the particularities of endoscopic instruments make possible a a less invasive technique compared to craniotomy: only a simple trepan hole and very small cortical incision. Stereotactic guided techniques frame- based or neuronavigaton are especially useful in patients without hydrocephalus.

Endoscopy allows the cyst puncture and aspiration under better visual control, the cyst wall resection with specific tools, and laser coagulation. The existence of a viewing field makes endoscopy superior to stereotactic aspiration. May be encountered difficulties related to the viscosity of cystic content, but they can be technically overcome with the price of increasing the operative time (8).

Cyst location and degree of distension of the third ventricle roof, with the emergence of a Monro foramen may cause problems when using a rigid endoscope, the view being impossible or difficult. Posterior interventricular foramen puncture may be helpful in such cases. Traction on adherent ceiling remains of the capsule may lead to bleeding of the ventricular blood vessels in 
the area. A major bleeding can blur the image and can be difficult to stop using the available specific tools. The major disadvantage of the technique is that not always the colloid cyst can be completely removed.

Although neuroendoscopic instrumentation development and the use of neuronavigation lowered the complication rate, endoscopic technique requires experience. A well trained surgical team obtaines promising results.

Only long-term prospective studies on the evolution of natural colloids cysts will demonstrate the need for treatment in asymptomatic cases. In symptomatic cases, endoscopic resection can should be considered as first intention when the cyst seems to have a liquid content and there ventriculomegaly is present (16). Endoscopic approach tends to replace stereotactic aspiration. Conventional surgery should be performed whenever endoscopy is not indicated or can not provide satisfactory results. Transcallosal approach is used especially in the absence of ventriculomegaly.

\section{Complications}

Although the development of endoscopic techniques and their application on a large scale has led to fewer complications, there is no doubt that an operative team requires some time for learning and gaining experience of all technical details. Specific morbidity rate is low $(11,15,18,20,23)$. Fornix lesions by the manipulation of instruments, thermal damage by excessive coagulation, intraoperative bleeding, aseptic or septic ventriculitis are potential complications of endoscopic surgery $(8,11,23,24,25)$. Overall morbidity is transient.
Bleeding can complicate endoscopic intervention. It is very important to prevent the bleeding, because even a slight hemorrhage may affect the visualization. In general, small hemorrhages spontaneously stop after saline solution irrigation. It should be noted that a minor capillary bleeding is best left to stop spontaneously, in order to form a clot that remains in situ under the influence of gravity. In this way we prevent the turbulence in the cerebrospinal fluid (26). Clots are removed at the end of the intervention by aspiration. A major bleeding is difficult to control endoscopically.

\section{Results}

Symptoms caused by intracranial hypertension usually resolves after surgery. Memory problems may persist some time.

Endoscopic resection of colloids cysts can be done as radical as microsurgical resection $(5,15,23)$. Although a complete excision is desirable, it should not be achieved with the cost of severe bleeding that can occur in firmly adherent ventricular cysts $(1,8,23)$. In such cases, the most prudent solution is the coagulation of the cystic wall . It seems that the existence of a small cystic remnant attached to the stroma of the choroid of the third ventricle does not involve a significant risk of clinical recurrence (11). The recurrence interval is usually long, so surveillance of endoscopic treated patients have achieved long periods (more than 10 years) before deciding that endocsopy should be a method of choice replacing microsurgery (23). The overall rate of recurrence is low, both clinical and radiological one (8). Many series have not recurring records $(1,13,15,18,20,23)$. 
DOI: $10.2478 / v 10282-012-0004-3$

It is important to note that after endoscopic interventions there are is not usually required drainage of cerebrospinal fluid, unlike microsurgical interventions, where in $10 \%$ of cases postoperative shunts are necessary. (14). A patient treated endoscopically is hospitalized for a period of $2-5$ days $(13,14,15,18,20)$, about $50 \%$ less than a microsurgery operated patient. Patients may return to work or their previous daily activities in the within 15 days (7-26 days) $(13,18,20)$.

\section{Conclusions}

So far, no consensus on optimal therapeutic strategy for colloids cyst has been reached, especially these tumors are slow-growing, non-invasive benign lesions. In symptomatic cases, total or near-total resection of colloid cysts can be achieved with endoscopy, with little morbidity, shortened operative time, reduced length of hospital stay and resolution of symptoms. Our experience suggests that rigid endoscopy is an excellent technique for surgical management of the colloid cysts. The endoscopic approach using modern instrumentation combined with framebased or frameless stereotactic guidance, allows complete or near complete removal of the cyst wall and should result in lower recurrence rate compared to microsurgery.

Technically speaking, difficulties may arise regarding the location of the cyst. Sometimes it causes an interventricular foramen and transforaminal approach becomes impossible. An alternative is the puncture of the tumoral mass posterior to the foramen of Monro, but there is a risk of intercepting the neighborhood veins.

\section{References}

1. Abdou MS, Cohen AR. Endoscopic treatment of colloid cysts in the third ventricle. Technical note and review of the literature. Journal of Neurosurgery 89: 1062-1068, 1998.

2. Antunes JL, Louis KM, Ganti SR. Colloid cysts of the third ventricle. Neurosurgery 7: 450-455, 1980.

3. Baskin JJ, Manwaring KH. Endoscopic excision of colloid cysts. In: Rengachary SS, Wilkins RH, ed. Neurosurgical operative atlas. Vol. 6: The American Association of Neurological Surgeons, pp. 109-114, 1997.

4. Batnitsky S, Sarwar M, Leeds NE, Schechter MM, Azar-Kiab M. Colloid cysts of the third ventricle. Radiology 112: 327-341, 1974.

5. Caemaert J, Abdullah J. Endoscopic management of colloid cysts. Tech Neurosurg 1: 185-200, 1996.

6. Crone KR, Miller MN. Colloid cysts: endoscopy vs. microsurgical treatment. In: King WA, Frazee JG, De Salles AAF, ed. Endoscopy of the central and peripheral nervous system. New York: Thieme, pp. 78-83, 1998.

5. Decq P, Le Guerinel C, Sakka L, Roujeau T, Sol JC, Palfi S, Nguyen JP. Approche endoscopique des lesions $\mathrm{du}$ troisieme ventricule. Neurochirurgie 46 (3): 286294, 2000.

8. Decq P, Le Guerinel C, Brugieres P, Djindjian M, Silva D, Keravel Y, Melon E, Nguyen JP. Endoscopic 9. Donaldson JO, Simon RH. Radiodense ions within a third ventricular colloid cyst. Arch Neurol 37: 246, 1980.

10. Hall WA, Lunsford LD. Changing concepts in the treatment of colloid cysts. Journal of Neurosurgery 66: 186-191, 1987.

11. Hellwig D BB, Schulte M, Gatscher S, Riegel T, Bertalanffy H. Neuroendoscopic treatment for colloid cysts of the third ventricle: the experience of a decade. Neurosurgery 52: 525-514, 2003.

12. Hernesniemi J, Leivo S. Management outcome in third ventricular colloid cysts in a defined population. A series of 40 patients treated mainly by transcallosal microsurgery. Surgical Neurology 45: 2-14, 1996.

13. Kahn EA, Crosby EC, De Jonge BR. Tumors of the diencephalon. In: Kahn EA, Crosby EC, Schneider RC et al, ed. Corelative Neurosurgery. Second ed. Springfield, Il: Charles C Thomas, pp. 131-153, 1969.

14. Kehler U BA, Gliemroth J, Nowak G, Delitala A, Chiappetta F, Arnold H. Twenty colloid cystscomparison of endoscopic and microsurgical management. Minimally Invasive Neurosurgery 44: 121-127, 2001.

15. King WA, Ullman JS, Frazee JG, Post KD, Bergsneider M. Endoscopic resection of colloid cysts: surgical consideration using the rigid endoscope. Neurosurgery 44 (5): 1103-1111, 1999. 
16. Le Gars D, Lejeune JP, Desenclos C. Tumeurs du troisieme ventricule: revue de la litterature. Neurochirurgie 46 (3): 296-319, 2000.

17. Laidlaw J, Kaye AH. Colloid cysts. In: Kaye AH, Laws Jr ER, ed. Brain tumors. An encyclopedic approach. London: Churchill Livingstone, pp. 983-996, 2001.

18. Lewis AI, Crone KR, Taha J, et al. Surgical resection of third ventricle colloid cysts. Preliminary results comparing transcalosal microsurgery with endoscopy. Journal of Neurosurgery 81: 174-178, 1994.

19. Little JR MC. Colloid cysts of the third ventricle. Journal of Neurosurgery 40: 230-235, 1974.

20. Longatti P MA, Moro M, Fiorindi A, Carteri A Endoscopic treatment of colloid cysts of the third ventricle: 9 consecutive cases. Minimally Invasive Neurosurgery 43: 118-123, 2000.

21. Mohadjer M, Teshmar E, Mundinger F. CTstereotaxic drainage of colloid cysts in the foramen of
Monro and the third ventricle. Journal of Neurosurgery 67: 220-223, 1987.

22. Sackett JF, Messina AV, Petito CK. Computed tomography and magnification vertebral angiotomography in the diagnosis of colloid cysts of the third ventricle. Radiology 116: 95-100, 1975.

23. Schroeder HWS GM. Endoscopic resection of colloid cysts. Neurosurgery 51: 1441-1445, 2002.

24. Teo C. Complete endoscopic removal of colloid cysts: issues of safety and efficacy. Neurosurg Focus 6 (4): e9, 1999.

25. Tirakotai W, Schulte DM, Bauer BL, Bertalanffy H, Hellwig D. Neuroendoscopic surgery of intracranial cysts in adults. Childs Nervous System 20 (11-12): 842851, 2004.

26. Torrens MJ. Endoscopy for ventricular lesions. In: Kaye AH, Black PM, ed. Operative Neurosurgery. Vol. 1. London: Churchill Livingstone, pp. 777-788, 2000. 